\title{
Article
}

\section{A Multi-Objective Collection-Distribution Center Location and Allocation Problem in a Closed-Loop Supply Chain for the Chinese Beer Industry}

\author{
Kai Kang, Xiaoyu Wang and Yanfang Ma * \\ School of Economics and Management, Hebei University of Technology, Tianjin 300401, China; \\ kkang2000@sina.com \\ * Correspondence: mayanfang@hebut.edu.cn; Tel.: +86-22-6043-5164
}

\begin{abstract}
Recycling waste products is an environmental-friendly activity that can bring benefits to accompany, saving manufacturing costs and improving economic efficiency. For the beer industry, recycling bottles can reduce manufacturing costs and reduce the industry's carbon footprint. This paper presents a model for a multi-objective collection-distribution center location and allocation problem in a closed loop supply chain for the beer industry, in which the objective is to minimize total costs and transportation pollution. Uncertainties in the form of randomness and fuzziness are jointly handled in this paper to ensure a more practical problem solution, for which returned bottle sand unusable bottles are considered fuzzy random variables. A heuristic algorithm based on priority-based global-local-neighbor particle swarm optimization (pb-glnPSO) is applied to ensure reliable solutions for this NP-hard problem. A case study on a beer operation company is conducted to illustrate the application of the proposed model and demonstrate the priority-based global-local-neighbor particle swarm optimization.
\end{abstract}

Keywords: collection-distribution center; closed loop supply chain; fuzzy random variable; particle swarm optimization

\section{Introduction}

Due to resource scarcity and environmental concerns, responsible companies are beginning to pay attention to the future of the planet and the global environment. Recycling used products for remanufacturing is, therefore, becoming of greater importance in supply chain management, a move that can dramatically reduce carbon emissions [1]. Closed loop supply chains (CLSC) combine the forward supply chain with a reverse supply chain that covers the whole life cycle of the products [2], with the manufacturing of new products and the transportation to customers via distribution centers and retailers as the forward supply chain and recycling, sorting, disposal and remanufacturing as the reverse supply chain. In recent years, the CLSC has received a great deal of academic and business attention because of the need to be socially responsible, environmental concerns and government legislation [3,4], which has motivated companies to pay more attention to recycling to reduce costs and lessen their carbon footprint.

Facility location and allocation problems (FLAP) have been widely studied. Subramanian [5] developed priority based simulated annealing to solve a CLSC network design problem, in which the distribution center (DC) and the centralized return center (CC) were set. Amin [6] presented a multi-objective facilities location model for manufacturing and remanufacturing plants and CLSC collection centers, which included demand and return uncertainties. Subulan [7] developed a multi-objective CLSC network design model for the lead/acid battery industry that considered both financial and collection objectives. CLSC network design in a competitive environment with price-dependent demand was examined by Rezapour [4], in which the DC and CC were built separately. Zeballos [8] proposed a model for a multi-period CLSC design and planning problem with demand uncertainty that had ten echelons in which the DC and CC were considered. Oh [9] developed a multi-objective model for profits and carbon emissions to determine optimal production, 
transportation and inventory quantities on a CLSC network in the fashion industry. Khatami [10] proposed a scenario-based stochastic mixed-integer linear programming model to solve a CLSC network design problem, in which the retailers' demand and the quantity of returned products were considered to be uncertain and the DC and CC were set. Vahdani [11] proposed capacitated bidirectional facilities to conduct distribution in a CLSC, in which a multi-priority queuing system was studied. Kim [12] designed a model to minimize the manufacturer's total cost to find the optimal solution to the supply of raw material, the quantity of products and materials to be recycled, the recycling facility scale and the potential benefits or downfalls of joining a recycling association. As a growing number of companies are now engaging in recycling activities due to economic and environmental concerns, distribution and collection activities using the same vehicle has been found to reduce carbon emissions and transportation costs because empty loads can be avoided. In this paper, we combine the distribution center (DC) with the collection center (CC) as a collection-distribution center (CDC), which can benefit company operations and reduce construction costs. In practice, as the recycled product owners are usually at the same location as the potential new product buyer [13], a DC/CC combination requires less construction and operating expense sand can significantly reduce environmental pollution.

Ramkumar [14] developed a multi echelon, multi period, multi product closed loop supply chain network model which was solved using a genetic algorithm with fixed variables. Kaya and Onur [13] presented a facilities location-inventory-pricing model without uncertainty to determine the optimal location for facilities. Barz [15] proposed an optimization model for a two-stage capacitated facilities location and allocation problem with the effects of additive manufacturing, in which all the variables were certain. Jindal [16] developed a multi-objective model for a CLSC network design problem that considered the economic and environmental factors as fuzzy uncertain and in which the DC and CC were separate. Ramezani [17] conducted research into a CLSC network design problem that only considered fuzzy variables. In recent years, uncertainty has attracted more research attention [18-20]. Stochastic programming, robust optimization, and fuzzy set theory are three applicable tools which can be used to present uncertainty in the FLAP [21,22]. Keyvanshokooh [23] proposed a novel hybrid robust-stochastic programming (HRSP) approach to simultaneously model two different types of uncertainties by including stochastic scenarios for transportation costs and polyhedral uncertainty sets for demands and returns. However, they considered the DC and the CC to be separate and the collection disposal rate was treated as a certain variable. Uncertainties exist in both the forward supply and reverse supply chains; however, the uncertainties in the reverse flow are higher than those in the forward supply chain $[7,19]$, with the returned product quantity generally being considered uncertain $[10,23]$. Subjective uncertainties such as decision maker's choices and the environmental coefficients can be dealt with using fuzziness and objective uncertainties such as unit transportation costs, product prices and the quantity of unusable products can be dealt with using randomness. In this paper, the return rate and disposal rate are considered fuzzy random variables to reflect the problem. The random and fuzzy uncertainties are handled together and represented by triangular fuzzy numbers [7]. Based on the above consideration, the model is formulated to determine the proper number and location of the CDCs as well as the allocation strategy between the different kinds of facilities.

Because of their structure, facilities location and allocation problems are non-convex and non-differentiable and are strongly NP-hard problems. A collection and distribution center location and allocation problem (CDCLAP) in a closed loop supply chain under a fuzzy random environment, therefore, is even more complicated. Particle swarm optimization(PSO) has been shown to be effective in solving NP-hard problems [24-26]. However, after observation, when the local optimal solution is found, the particles' behavior in the basic PSO is directly influenced, which means that it frequently falls into a local optimum [27-29]. Different advanced PSOs have been used to solve supply chain management problems. Ai and Kachitvichyanukul [30] proposed a global-local-neighbor PSO which was more effective, based on which $\mathrm{Xu}$ [27] proposed a fuzzy 
random simulation-based bi-level global-local-neighbor particle swarm optimization (frs-bglnPSO). In this paper, a priority-based global-local-neighbor particle swarm optimization (pb-glnPSO) is applied to solve the CDCLAP.

In summary, this paper proposes a multi-objective model to solve a collection-distribution center location and allocation problem in a closed loop supply chain that considers the economic and environmental factors and includes fuzzy random variables for the return and disposal rates. The remainder of this paper is organized as follows: Section 2 presents the problem statement and model assumptions. A description of the model and its formulation are given in Section 3. The proposed hybrid solution based on the pb-glnPSO is described in Section 4. A case study is conducted to illustrate the model formulation and the proposed method in Section 5. Finally, Section 6 concludes this paper.

\section{Research problem statement}

In this paper, a company with factories at certain locations and several retailers at different customer zones are considered. The company is considering whereto set the integrated collection and distribution centers (CDC), at which both the collection network for used products and the distribution network for new products are jointly established [13]. CDCs reduce both construction and transportation costs because the same vehicles can be used for both distribution and recycling. Therefore, in this paper, only CDCs are considered.

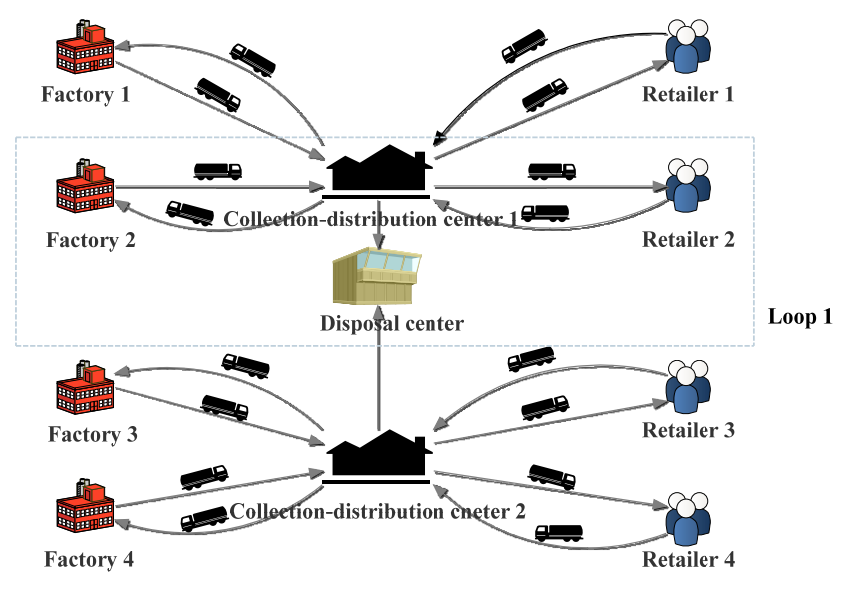

Figure 1. The closed loop supply chain network

A general illustration of the classical CDCLAP for a closed loop supply chain is shown in Fig 1, with the CLSC framework shown in loop1. The CLSC framework has four echelons: factories, CDCs, retailers and disposal centers [11]. The forward supply chain begins with new production. From the factories, the finished products are transported to the retailers via the CDCs. In the reverse supply chain, the returned products are collected and transported to the CDCs, where the recycled products are inspected, consolidated and sorted into those that are available for remanufacturing, which are sent to the factories, and those that are unsuitable for remanufacturing, which are transported to the disposal centers [23]. A CDC can supply products to multiple retailers and retailer demand is fulfilled by only one production site. A CDC can handle products from different factories and send the returned products to multiple factories for remanufacturing.

In this CLSC, the retailers' demand is estimated based on pre-orders; however, the return rate is considered fuzzy random as customers may not return the used product or the product may have broken. In consideration of the transportation costs and the carrying loss, the availability of recycled products is unsure. Transportation costs and transportation pollution are related to the distance 
between two facilities. Another fuzzy random variable considered in this paper is the returned product disposal rate, which is decided on after inspection and consolidation at the CDC.

Following are the assumptions in the proposed problem investigation: (1) Only one product one period is considered; (2) All alternative locations for the CDCs have been identified; (3) Recycling a used product costs less than manufacturing a new one [31]; (4) Considering uncapacitated facilities is an unrealistic assumption in many LAP problems. Many researchers assign a maximum capacity level to facilities to model more realistic decisions. The CDCs and the factories have a capability limit [32-34]; (5) The locations for the factories, retailers and disposal centers are known; (6) New product and returned product storage is allowed at the CDCs [22].

The initial problem is making a decision as to where to set the CDCs from the candidate sites and deciding on an allocation strategy at minimal total CDC costs; operating costs, transportation costs and transportation pollution cost; while also considering the flow constraints, capability limits and the retailers' demand.

\section{Modelling}

In this section, a mathematical description is given for the CDCLAP in the CLSC, including the notations, the research problem statement, and the mathematical formulation.

\subsection{Notations}

To facilitate the problem description, the notations are explained.

Sets

$\Omega$ : set of CDCs, $\Omega=\{1,2,3, \ldots, I\}$.

$\Psi$ : set of factories, and $\Psi=\{1,2,3, \ldots, J\}$

$\Phi$ : set of retailers, and $\Phi=\{1,2,3, \ldots, K\}$

$Y$ : set of disposal centers, and $Y=\{1,2,3, \ldots, N\}$

Indices and parameters

$i$ : alternative location position for the CDCs, $i \in \Omega=\{1,2,3, \ldots, I\}$.

$j$ : known position of the factories, $j \in \Psi=\{1,2,3, \ldots, J\}$.

$k$ : known position of the retailers, $k \in \Phi=\{1,2,3, \ldots, K\}$.

$n$ : known disposal center, $n \in \mathrm{Y}=\{1,2,3, \ldots, N\}$.

$U$ : the upper limit of the CDCs.

$D_{k}$ : the demand of retailer $k$.

$\alpha_{i}$ : the capability of CDC $i$.

$\gamma_{j}$ : the capability of factory $j$.

$P_{j i}$ : product quantity from factory $j$ to CDC $i$.

$Q_{i k}$ : product quantity from CDC $i$ to retailer $k$.

$\widetilde{\bar{a}}_{k}$ : the product return rate from retailer $k$.

$\widetilde{\bar{b}}_{i}$ : the product disposal rate at $\mathrm{CDC} i$.

$F_{i}^{c}$ : the fixed costs of the CDC.

$V_{i}^{c}$ : the variable cost of the CDC for a new product unit.

$R V_{i}^{c}$ : the variable cost of the CDC triage for a returned product unit.

$C_{i j}^{p}$ : unit transportation cost between CDC $i$ and factory $j$.

$C_{i k}^{d}$ : unit transportation cost between CDC $i$ and retailer $k$.

$C_{i n}^{w}$ : unit transportation cost between CDC $i$ and disposal center $n$.

$\beta_{i j}$ : environmental impact of transportation between CDC I and factory $j$.

$\beta_{i k}$ : environmental impact of transportation between CDC $i$ and retailer $k$.

$\beta_{\text {in }}$ : environmental impact of transportation between CDC $i$ and disposal center $n$. 


\section{Decision variables}

$x_{i}$ : a binary variable indicating whether point $i$ is chosen. If point $i$ is chosen, then $x_{i}=1$; else, $x_{i}=0$.

$y_{i k}$ : indicates whether retailer $k$ is served by CDC $i$. If $i$ is chosen, then $y_{i k}=1$; else, $y_{i k}=0$.

\subsection{Objective functions}

A multi-objective DCCLAP model using the above variables is proposed to minimize total costs and the environmental transportation effects.

Economic objective: In general, decision makers seek to minimize the total costs, which are made up of the transportation costs, fixed costs and operating costs. The minimization objective can be described as

$$
\begin{aligned}
\min Z_{1}= & \sum_{i=1}^{I} \sum_{j=1}^{I} C_{i j}^{p} P_{j i}+\sum_{i=1}^{I} \sum_{k=1}^{K} C_{i k}^{d} Q_{i k}\left(1+\widetilde{\bar{a}}_{k}\right)+\sum_{i=1}^{I} \sum_{n=1}^{N} \sum_{k=1}^{K} C_{i n}^{w} \widetilde{\bar{b}}_{i} \widetilde{\bar{a}}_{k} Q_{i k}+\sum_{i=1}^{I} \sum_{j=1}^{J} \sum_{k=1}^{K} \sum_{n=1}^{N} C_{i j}^{p} \widetilde{\bar{a}}_{k} Q_{i k}\left(1-\widetilde{\bar{b}}_{i}\right) \\
& +\sum_{i=1}^{I} F_{i}^{c} X_{i}+\sum_{i=1}^{I} \sum_{j=1}^{J} V_{i}^{c} P_{j i}+\sum_{i=1}^{I} \sum_{k=1}^{K} R V_{i}^{c} \widetilde{\bar{a}}_{k}
\end{aligned}
$$

Equation (1) calculates the total cost, in which $\sum_{i=1}^{I} \sum_{j=1}^{J} C_{i j}^{p} P_{j i}$ represents the cost of new product transported from factories to $\mathrm{CDC}, \sum_{i=1}^{I} \sum_{k=1}^{K} C_{i k}^{d} Q_{i k}\left(1+\overline{\bar{a}}_{k}\right)$ calculated the transportation cost between CDCs and retailers, $\sum_{i=1}^{I} \sum_{n=1}^{N} \sum_{k=1}^{K} C_{i n}^{i v} \widetilde{\bar{b}}_{i} \widetilde{\bar{a}}_{k} Q_{i k}$ is the cost of returned product delivered from CDCs to disposal centers as well as the returned product transportation cost from CDCs to disposal centers is measured as $\sum_{i=1}^{I} \sum_{j=1}^{J} \sum_{k=1}^{K} \sum_{n=1}^{N} C_{i j}^{p} \widetilde{\bar{a}}_{k} Q_{i k}\left(1-\widetilde{\bar{b}}_{i}\right)$. The fixed cost of opening a new CDC is presented as $\sum_{i=1}^{I} F_{i}^{c} X_{i} . \sum_{i=1}^{I} \sum_{j=1}^{J} V_{i}^{c} P_{j i}$ shows the variable cost of new product. $\sum_{i=1}^{I} \sum_{k=1}^{K} R V_{i}^{c} \overline{\bar{a}}_{k}$ calculates the operation cost of returned product.

It is very difficult to handle the objective function with fuzzy random factors. Kruse and Meyer [35] point out that the fuzzy expected value may be represented by a single fuzzy number. Without a loss of generality, based on the theory proposed by Heilpern [36], the expected value operator is used to enable the conversion of the uncertain model into the deterministic. Now the fuzzy random objective function can be transformed into their crisp equivalences as shown in Eq. (2):

$$
\begin{aligned}
\min Z_{1}= & \sum_{i=1}^{I} \sum_{j=1}^{I} C_{i j}^{p} P_{j i}+\sum_{i=1}^{I} \sum_{k=1}^{K} C_{i k}^{d} Q_{i k}\left(1+E V\left[\tilde{\bar{a}}_{k}\right]\right)+\sum_{i=1}^{I} \sum_{n=1}^{N} \sum_{k=1}^{K} C_{i n}^{w} E V\left[\tilde{\bar{b}}_{i}\right] E V\left[\widetilde{\bar{a}}_{k}\right] Q_{i k} \\
& +\sum_{i=1}^{I} \sum_{j=1}^{I} \sum_{k=1}^{K} \sum_{n=1}^{N} C_{i j}^{p} E V\left[\tilde{\bar{a}}_{k}\right] Q_{i k}\left(1-E V\left[\tilde{\bar{b}}_{i}\right]\right)+\sum_{i=1}^{I} F_{i}^{c} X_{i}+\sum_{i=1}^{I} \sum_{j=1}^{J} V_{i}^{c} P_{j i}+\sum_{i=1}^{I} \sum_{k=1}^{K} R V_{i}^{c} E V\left[\widetilde{\bar{a}}_{k}\right]
\end{aligned}
$$

Note the $E V\left[\tilde{\bar{a}}_{k}\right]$ or $E V\left[\widetilde{\bar{b}}_{i}\right]$ above represents two expected values: the first one being the fuzzy random variables converted into fuzzy numbers based on the theory proposed by Kruse and Meyer in 1987, and the second being used to transform the fuzzy numbers into deterministic numbers based on the theory proposed by Heilpern in 1992.

Environmental objective: The second objective is to minimize the environmental transportation effect in terms of the carbon emissions in the CLSC operation, an area which has attracted recent research attention [37]. The following expression represents the transportation carbon emissions between the CDCs and the factories, the CDCs and the retailers and the CDCs and the disposal centers. 


$$
\begin{aligned}
\min Z_{2}= & \sum_{i=1}^{I} \sum_{j=1}^{J} \beta_{i j} P_{j i}+\sum_{i=1}^{I} \sum_{k=1}^{K} \beta_{i k} Q_{i k}\left(1+E V\left[\widetilde{\bar{a}}_{k}\right]\right)+\sum_{i=1}^{I} \sum_{n=1}^{N} \sum_{k=1}^{K} \beta_{i n} E V\left[\widetilde{\bar{b}}_{i}\right] E V\left[\widetilde{\bar{a}}_{k}\right] Q_{i k} \\
& +\sum_{i=1}^{I} \sum_{j=1}^{J} \sum_{k=1}^{K} \sum_{n=1}^{N} \beta_{i j} E V\left[\widetilde{\bar{a}}_{k}\right] Q_{i k}\left(1-E V\left[\widetilde{\bar{b}}_{i}\right]\right)
\end{aligned}
$$

$\sum_{i=1}^{I} \sum_{j=1}^{J} \beta_{i j} P_{j i}$ refers to the environment pollution caused by transportation activities from factories to CDCs. $\sum_{i=1}^{I} \sum_{k=1}^{K} \beta_{i k} Q_{i k}\left(1+E V\left[\tilde{\bar{a}}_{k}\right]\right)$ is the summation of carbon footprints when transporting products between CDCs and retailers. $\sum_{i=1}^{I} \sum_{n=1}^{N} \sum_{k=1}^{K} \beta_{i n} E V\left[\overline{\bar{b}}_{i}\right]$

$E V\left[\tilde{\bar{a}}_{k i}\right] Q_{i k}$ is the total carbon footprint from CDCs to disposal centers. And $\sum_{i=1}^{I} \sum_{j=1}^{J} \sum_{k=1}^{K} \sum_{n=1}^{N} \beta_{i j} E V\left[\widetilde{\bar{a}}_{k}\right] Q_{i k}\left(1-E V\left[\overline{\bar{b}}_{i}\right]\right)$ express the carbon footprints from CDCs to factories when delivering returned products.

\subsection{Constraints}

Note that the CDC has its own capacity limit and it cannot service any goods beyond its capacity. Thus we need capacity restriction. The constraint can be written as follows:

$$
\sum_{k=1}^{K} E V\left[\widetilde{\bar{a}}_{k}\right] Q_{i k}+\sum_{j=1}^{J} P_{j i} \leq \alpha_{i} \quad \forall i \in \Omega
$$

$\widetilde{a_{k i}}$ is a fuzzy random variable indicating the return rate of the used product to transport from retailer $k$ to CDC $i$. $Q_{i k}$ shows product quantity from CDC $i$ to the retailer $k . P_{j i}$ indicates the quantity of product to transport from factory $j$ to CDC $i . \alpha_{i}$ refers to the capacity of the the capability of the CDC $i$.

As for capability constraint, the factory can manufacturing the new products that the retailers need and the returned products send back by CDCs.

$$
\sum_{k=1}^{K} D_{k}+\sum_{i=1}^{I} \sum_{k=1}^{K} \sum_{n=1}^{N} C_{i j}^{p} E V\left[\widetilde{\bar{a}}_{k}\right] Q_{i k}\left(1-E V\left[\widetilde{\bar{b}}_{i}\right]\right) \leq \sum_{j=1}^{J} \gamma_{j}
$$

$D_{k}$ refers to the demand of retailer $k$ and $\gamma_{j}$ is the capability of the factory $j$. $\sum_{i=1}^{I} \sum_{k=1}^{K} \sum_{n=1}^{N} C_{i j}^{p} E V\left[\widetilde{\bar{a}}_{k}\right] Q_{i k}\left(1-E V\left[\widetilde{\bar{b}}_{i}\right]\right)$ calculates the returned product transported to factory $j$ for remanufacture.

Considering the products in the retailers are all from the CDCs, the recycled products are less than the product transported from factory to the CDC And it can be described as follows:

$$
\sum_{j=1}^{J} P_{j i} \geq \sum_{k=1}^{K} E V\left[\widetilde{\bar{a}}_{k}\right] Q_{i k}
$$

$P_{j i}$ is a variable indicating the quantity of new product transported from the factory $j$ to CDC $i . \tilde{\overline{a_{k}}} Q_{i k}$ refers to the quantity of returned product transported from retailer $k$ to CDC $i$.

The product provided to the retailer should at least meet the retailer's demand.

$$
\sum_{i=1}^{I} Q_{i k} \geq \sum_{k=1}^{K} D_{k}
$$

$Q_{i k}$ indicates the product quantity CDC $i$ to the retailer $k$. the stochastic variable $D_{k}$ is the demand of the retailer $k$ according to the order. 
The returned products transported to the CDCs are more than the products transported to the disposal centers.

$$
\sum_{k=1}^{K} E V\left[\widetilde{\bar{a}}_{k}\right] Q_{i k} \geq \sum_{n=1}^{N} \sum_{k=1}^{K} E V\left[\tilde{\bar{b}}_{i}\right] E V\left[\widetilde{\bar{a}}_{k}\right] Q_{i k}
$$

$E V\left[\tilde{\bar{a}}_{k}\right] Q_{i k}$ is the expression of returned product quantity from the retailer $k$ to $\operatorname{CDC} i, E V\left[\widetilde{\bar{b}}_{i}\right] E V\left[\tilde{\bar{a}}_{k}\right] Q_{i k}$ presents the product quantity transported from CDC $i$ to retailer $k$.

The CDC should be at least one but no more than the upper limit.

$$
\begin{aligned}
& 1 \leq \sum_{i=1}^{I} x_{i} \\
& \sum_{i=1}^{I} x_{i} \leq U
\end{aligned}
$$

$\mathrm{U}$ is the upper limit of the CDCs, which is decided by the demand, the returned product quantity and the fixed capability.

It should make sure that each retailer is served by one CDC.

$$
\sum_{i=1}^{I} y_{i k}=1
$$

Since $x_{i}$ and $y_{i k}$ are binary variables, the following constraints are needed:

$$
\begin{aligned}
& x_{i}=\{0,1\}, \quad \forall i \in \Omega, \\
& y_{i k}=\{0,1\}, \quad \forall i \in \Omega, \quad \forall k \in \Phi
\end{aligned}
$$

$x_{i}$ is a binary variable indicating whether a CDC is opened at point $i$. If location $i$ is chosen to open a $\mathrm{CDC}$, then $x_{i}=1$; otherwise, $x_{i}=0 . y_{i k}$ is a binary variable indicating whether retailer $k$ is served by CDC $i$. If $y_{i k}=1$, then retailer $k$ is served by CDC $i$; otherwise, $y_{i k}=0$.

\subsection{Global model}

From the formulation above, a multi-objective model for the CDCLAP with capacity, flow and quantity constraints is developed with the aims of minimizing total costs and total transportation pollution. In the CLSC, both new and returned products are considered. The product can be reproduced to save raw materials and reduce waste and pollution. In our model, all costs involved in the CDCLAP are considered as well as the influence of the transportation activity pollution. Fuzzy random theory is used to deal with the real world complex uncertainties and ensure more scientific decisions. Therefore, this CDC situation is closer to the real situation as it can deal with complicated practical problems. Finally, the global model is given: 


$$
\begin{aligned}
& \min Z_{1}=\sum_{i=1}^{I} \sum_{j=1}^{J} C_{i j}^{p} P_{j i}+\sum_{i=1}^{I} \sum_{k=1}^{K} C_{i k}^{d} Q_{i k}\left(1+E V\left[\widetilde{\bar{a}}_{k}\right]\right)+\sum_{i=1}^{I} \sum_{n=1}^{N} \sum_{k=1}^{K} C_{i n}^{w} E V\left[\widetilde{\bar{b}}_{i}\right] E V\left[\widetilde{\bar{a}}_{k}\right] Q_{i k} \\
& +\sum_{i=1}^{I} \sum_{j=1}^{J} \sum_{k=1}^{K} \sum_{n=1}^{N} C_{i j}^{p} E V\left[\widetilde{\bar{a}}_{k}\right] Q_{i k}\left(1-E V\left[\tilde{\bar{b}}_{i}\right]\right)+\sum_{i=1}^{I} F_{i}^{c} X_{i}+\sum_{i=1}^{I} \sum_{j=1}^{J} V_{i}^{c} P_{j i}+\sum_{i=1}^{I} \sum_{k=1}^{K} R V_{i}^{c} E V\left[\widetilde{\bar{a}}_{k}\right] \\
& \min Z_{2}=\sum_{i=1}^{I} \sum_{j=1}^{J} \beta_{i j} P_{j i}+\sum_{i=1}^{I} \sum_{k=1}^{K} \beta_{i k}\left(Q_{i k}+E V\left[\tilde{\bar{a}}_{k}\right]\right)+\sum_{i=1}^{I} \sum_{n=1}^{N} \beta_{i n} E V\left[\tilde{\bar{b}}_{i}\right]+\sum_{i=1}^{I} \sum_{j=1}^{J} \sum_{k=1}^{K} \sum_{n=1}^{N} \beta_{i j}\left(E V\left[\widetilde{\bar{a}}_{k}\right]-E V\left[\widetilde{\bar{b}}_{i}\right]\right) \\
& \left\{\begin{array}{l}
\sum_{k=1}^{K} E V\left[\widetilde{\bar{a}}_{k i}\right] Q_{i k}+\sum_{j=1}^{J} P_{j i} \leq \alpha_{i} x_{i} \quad \forall i \in \Omega \quad \forall j \in \Psi \quad \forall k \in \Phi \\
\sum_{k=1}^{K} D_{k}+\sum_{i=1}^{I} \sum_{k=1}^{K} \sum_{n=1}^{N} C_{i j}^{p} E V\left[\widetilde{\bar{a}}_{k}\right] Q_{i k}\left(1-E V\left[\widetilde{\bar{b}}_{i}\right]\right) \leq \sum_{j=1}^{J} \gamma_{j} \quad \forall j \in \Psi \quad \forall k \in \Phi \quad \forall n \in Y
\end{array}\right. \\
& \sum_{j=1}^{J} P_{j i} \geq \sum_{k=1}^{K} E V\left[\widetilde{\bar{a}}_{k i}\right] \quad \forall i \in \Omega \quad \forall j \in \Psi \quad \forall k \in \Phi \\
& \text { s.t. }\left\{\begin{array}{l}
\sum_{k=1}^{K} E V\left[\widetilde{\bar{a}}_{k}\right] Q_{i k} \geq \sum_{n=1}^{N} \sum_{k=1}^{K} E V\left[\widetilde{\bar{b}}_{i}\right] E V\left[\widetilde{\bar{a}}_{k}\right] Q_{i k} \quad \forall i \in \Omega \quad \forall k \in \Phi \quad \forall n \in \mathrm{Y} \\
\sum_{i=1}^{I} Q_{i k} \geq \sum_{k=1}^{K} D_{k} \quad \forall i \in \Omega \quad \forall k \in \Phi
\end{array}\right. \\
& 1 \leq \sum_{i=1}^{I} x_{i} \quad \forall i \in \Omega \\
& \sum_{i=1}^{I} x_{i} \leq U \quad \forall i \in \Omega \\
& y_{i k} \leq x_{i} \quad \forall i \in \Omega, \quad \forall k \in \Phi \\
& \sum_{i=1}^{I} y_{i k}=1 \quad \forall i \in \Omega \quad \forall k \in \Phi \\
& x_{i}=\{0,1\} \quad \forall i \in \Omega \text {, } \\
& y_{i k}=\{0,1\} \quad \forall i \in \Omega, \quad \forall k \in \Phi
\end{aligned}
$$

\section{The heuristic algorithms based on Pgln-PSO}

Particle swarm optimization (PSO) is a recent evolutionary algorithm which simulates social behavior such as birds flocking and fish schooling [38]. The PSO searches the feasible zone to seek solutions using a fixed population of individuals, which are updated to achieve the optimal solution. The particles [39] are characterized by their position and velocity, which are decided on by their flying experience or discoveries or those of their companions. They fly through the problem spaces following the current optimum particles to find the best solution between the populations and the best solution for each population. The PSO has been widely used to solve NP-hard problems [38]. However, in the basic PSO, it was found that the particles in the swarm were weak and clustered rapidly toward the global best particle [25]. Global-local-neighbor particle swarm optimization (glnPSO) proposed by Ai and Kachitvichyanukul [30] improves the weakness of the basic PSO. Xu and Yan [40] proposed a global-local-neighbor particle swarm optimization with exchangeable particles (GLNPSO-ep), which was even more advanced. In this section, a priority-based global-local-neighbor particle swarm optimization (pb-glnPSO) is proposed to solve the multi-objective CDCLAP in the CLSC.

\subsection{Notations for the Pb-glnPSO}

The basic elements of the PSO are particles, population, velocity, inertia weight, individual best and global best. The notations needed for the pb-glnPSO are as follows: 
$\tau: \quad$ iteration index, and $\tau=1,2, \ldots T$.

$d: \quad$ dimension index, and $d=1,2, \ldots D$.

$l: \quad$ particle index, and $l=1,2, \ldots L$.

$\omega_{\tau}$ : inertia weight in $\tau-t h$ iteration.

$v_{l d}(\tau): \quad$ velocity of the $l$ th particle at the $d$ th dimension in the $\tau$ th iteration.

$p_{d}^{l}(\tau)$ : $\quad$ position of the $l$ th particle at the $d$ th dimension in the $\tau$ th iteration.

$p_{l d}^{b e s t}: \quad$ personal best position.

$p_{g d}^{\text {best }}: \quad$ global best position.

$p_{\text {ld }}^{\text {Lbest }}$ : local best position.

$p_{l d}^{\text {Nbest: }} \quad$ near neighbor best position.

$c_{p}: \quad$ personal best position acceleration constant.

$c_{g}: \quad$ global best position acceleration constant.

$c_{l}: \quad$ local best position acceleration constant.

$c_{n}: \quad$ near neighbor best position acceleration constant.

$P^{\max }: \quad \quad$ maximum position value.

$P^{\text {min }} \quad \quad \quad$ minimum position value.

$P_{l}: \quad \quad$ velocity vector of $l$-th particle.

$V_{l}: \quad$ position vector of $l$-th particle.

$P_{l}^{\text {best }} \quad \quad \quad \quad$ vector personal best position of $l$-th particle.

$P_{g}^{b e s t}$ : $\quad$ vector global personal best position.

$P_{l}^{\text {Lbest }}$ : $\quad$ vector local best position of $l$-th particle.

$r_{1}, r_{2}, r_{3}, r_{4}:$ uniform distributed random number within [0,1].

Fitness $\left(P_{l}\right)$ : fitness value of $P_{l}$.

\subsection{Encoding and decoding algorithm}

The decoding process is based on the priority-based encoding developed by Gen and Cheng and the priority-based decoding and encoding proposed by Gen and Altiparmak [41]. The priorities of the CDCs and the retailers are equal to the total number of retailers and CDCs. At each step, the $\mathrm{CDC}$ (retailer) with the highest priority is selected and connected to a retailer (CDC) under a minimum transportation cost constraint. Table 1 shows the decoding algorithm for the priority-based encoding and its trace table, with the priority-based encoding considered random. The CDCLAP is solved in two stages [15]. In the first stage, the location for the CDCs is chosen and the transportation between the CDCs and the retailers calculated, while the second stage deals with the allocations between the factories and CDCs. 
Table 1. Decoding for the location and allocation problem

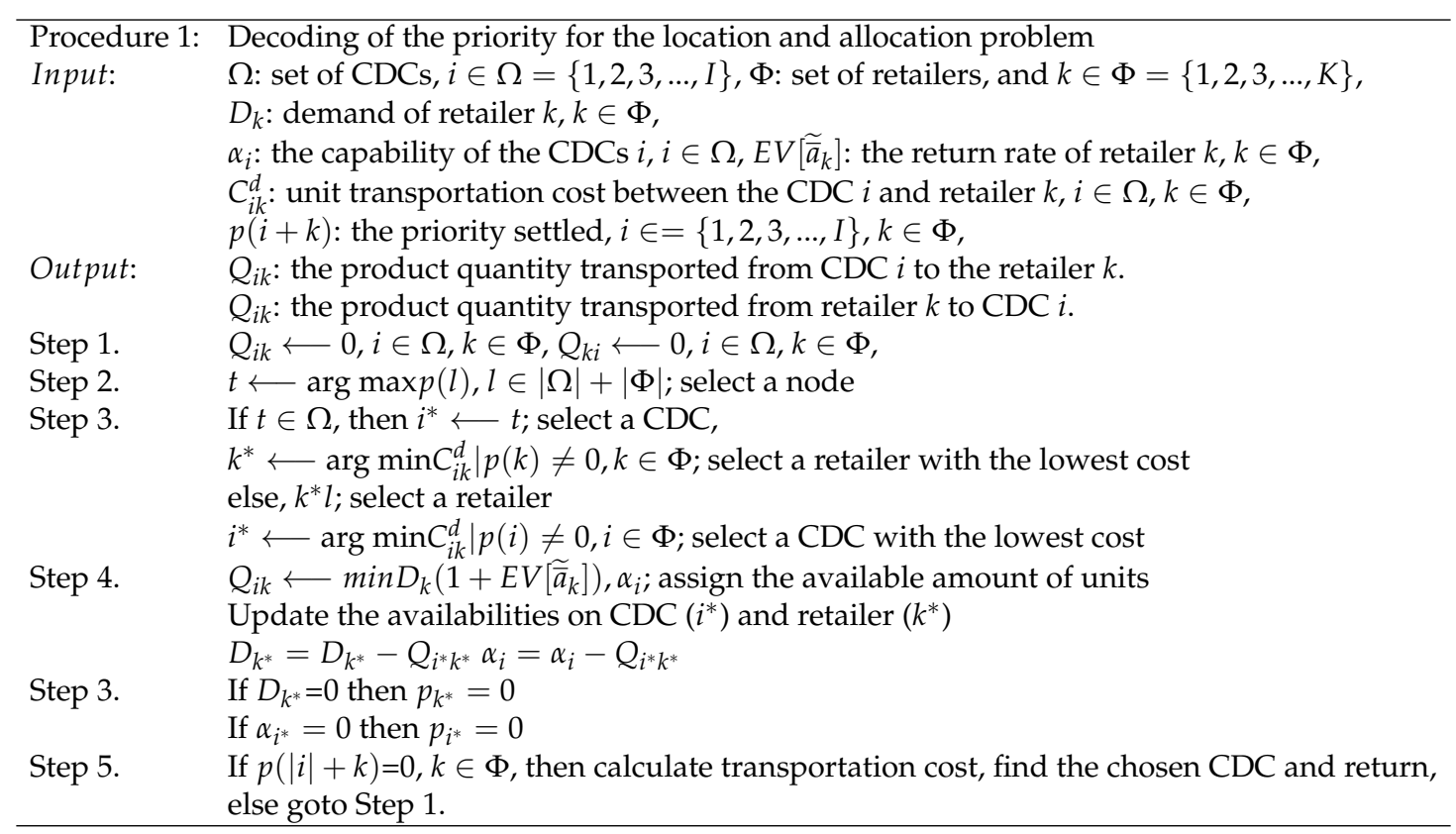

\subsection{Update}

Based on the above notations and the glnPSO proposed by Ai and Kachitvichyanukul [30], the inertia weight, velocity and position are updated using the following Equation.

$$
\begin{aligned}
& \omega(\tau)=\omega(T)+\frac{\tau-T}{1-T}[\omega(1)-\omega(T)] \\
& \begin{aligned}
v_{d}^{l}(\tau+1)= & \omega(\tau) v_{d}^{l}(\tau)+c_{p} r_{1}\left[p_{l d}^{\text {best }}(\tau)-p_{d}^{l}(\tau)\right]+c_{g} r_{2}\left[p_{g d}^{\text {best }}(\tau)-p_{d}^{l}(\tau)\right]+c_{l} r_{3}\left[p_{g d}^{\text {best }}(\tau)-p_{d}^{l}(\tau)\right] \\
& +c_{n} r_{4}\left[p_{g d}^{\text {best }}(\tau)-p_{d}^{l}(\tau)\right]
\end{aligned} \\
& p_{d}^{l}(\tau+1)=p_{d}^{l}(\tau)+v_{d}^{l}(\tau+1)
\end{aligned}
$$

The glnPSO has been widely used in solving NP-hard facilities location and allocation problems. 


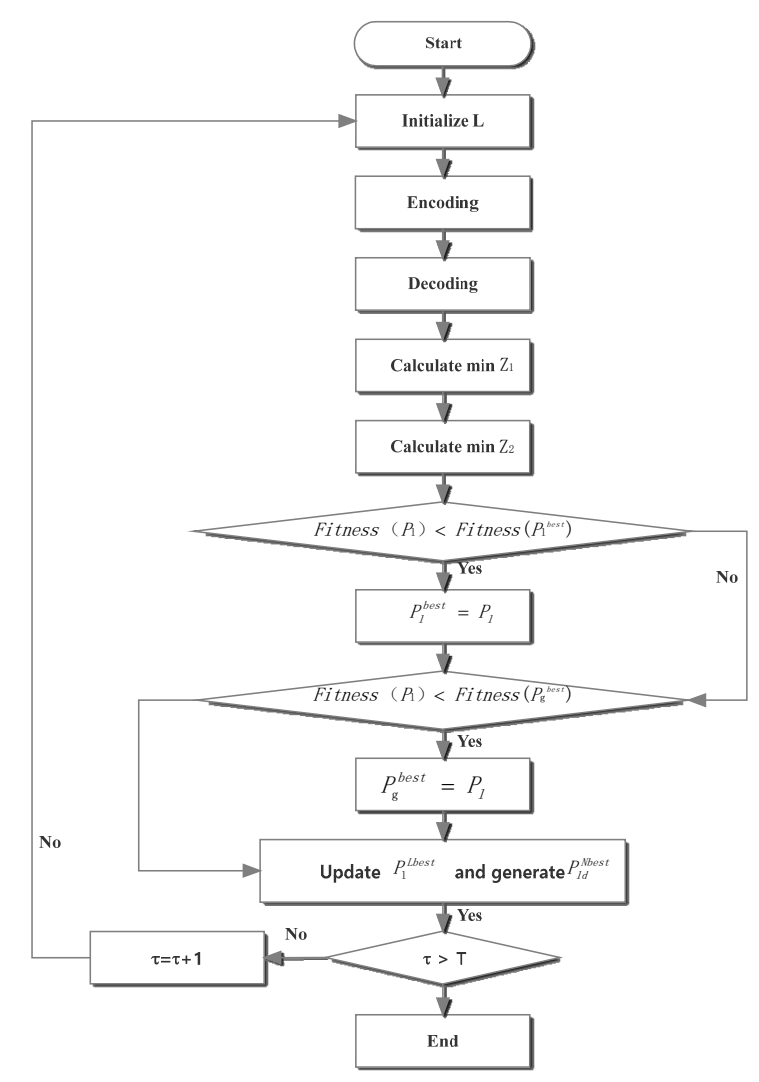

Figure 2. The heuristic algorithms based on pb-glnPSO

\subsection{Overall process of the $p b$-glnPSO}

In this paper, the glnPSO presented above is used to solve the location and allocation problem. Due to uncertainties and environmental changes, a priority-based global-local-neighbor particle swarm optimization (pb-glnPSO) is proposed to solve this model. As the company pays close attention to the economic costs, the environmental factor is dealt with as a constraint which has upper limits. The algorithmic details are as follows.

Step 1: Initialize P particles as a swarm: $l=1, \ldots L$, (the particle is the priority).

Step 2: Constraints check. If in the feasible region, goto step 3; otherwise, return to step 1.

Step 3: Calculate the fitness according to the decoding algorithm in Table 1.

Step 4: Update the particle positions and velocities.

Step 4.1: Acquire the expected value for $Z$ from the above algorithm.

Step 4.2: For $l=1,2, \ldots L$, decode each particle to an installment group. Calculate the fitness value of each particle and set as the position of the 1-th particle as its personal best. The global best position is chosen from these personal best positions.

Step 4.3: Update pbest: For $l=1,2, \ldots L$, if Fitness $\left(P_{l}\right)<$ Fitness $\left(P_{l}^{\text {best }}\right), P_{l}^{\text {best }}=P_{l}$.

Step 4.4: Update gbest: For $l=1,2, \ldots L$, if Fitness $\left(P_{l}\right)<$ Fitness $\left(P_{g}^{\text {best }}\right), P_{g}^{b e s t}=P_{l}$.

Step 4.5: Update lbest: For $l=1,2, \ldots L$, among all pbest of $M$ neighbors around the 1-th particle, set the personal best which has the best fitness value as $P_{l}^{\text {Lbest }}$.

Step 4.6: Generate nbest: For $l=1,2, \ldots L$, and $d=1,2, \ldots D$, find the $p_{o d}$ ensuring that the FDR takes a maximum value, and set $p_{o d}$ as $P_{l d}^{\text {Nbest }}$.

Step 4.7: Update the position and the velocity of each 1-th particle using Equation (18).

Step 4.8: Check whether the particles are beyond the mark. If $p_{l d}>P^{\max }$, the $p_{l d}=P^{\max }$; otherwise, if $p_{l d}<P^{\text {min }}$, thenn $p_{l d}=P^{\text {min }}$.

Step 5: Based on the above calculation, replace the ranking vector using the new numbers. 
Step 6: If the stopping criterion is met, stop; otherwise, $\tau=\tau+1$ and return to step 2. The overall process can be clearly seen in Fig 2.

\section{Case Study}

\subsection{Case presentation}

This model is motivated by a beer company in a developing country that bottles beer in plastic or glass bottles. The supply chain intends to allow customers to return the bottles to the retailers after the beer has been consumed, after which the returned bottles are sent to the CDCs where they are inspected, consolidated and sorted. After processing and disinfecting, the bottles are filled with beer and sold again. Now the company is considering the construction of several CDCs to allow for bottle recycling as producing a new bottle is far more expensive than recycling a used bottle.

Table 2. CDCs information (unit: $1 \times 10^{2} \mathrm{RMB}$ )

\begin{tabular}{cccccccc}
\hline Node & location & capability & fixed cost & NPV cost & RPV cost & $\overline{\bar{b}}_{i}$ & Parameters $\bar{\rho}$ \\
\hline 1 & $(23,23)$ & 900 & 12300 & 0.01 & 0.05 & $\left(0.18, \bar{\rho}_{1}, 0.25\right)$ & $\bar{\rho}_{1} \sim N(0.21,0.02)$ \\
2 & $(25,35)$ & 550 & 12100 & 0.02 & 0.06 & $\left(0.23, \bar{\rho}_{2}, 0.28\right)$ & $\bar{\rho}_{2} \sim N(0.25,0.02)$ \\
3 & $(34,29)$ & 1050 & 15600 & 0.01 & 0.05 & $\left(0.14, \bar{\rho}_{3}, 0.24\right)$ & $\bar{\rho}_{3} \sim N(0.18,0.04)$ \\
4 & $(32,25)$ & 650 & 11300 & 0.01 & 0.07 & $\left(0.16, \bar{\rho}_{4}, 0.22\right)$ & $\bar{\rho}_{4} \sim N(0.18,0.03)$ \\
5 & $(35,37)$ & 1050 & 17800 & 0.01 & 0.05 & $\left(0.25, \bar{\rho}_{5}, 0.30\right)$ & $\bar{\rho}_{5} \sim N(0.28,0.02)$ \\
6 & $(36,31)$ & 1050 & 22400 & 0.01 & 0.06 & $\left(0.17, \bar{\rho}_{6}, 0.26\right)$ & $\bar{\rho}_{6} \sim N(0.22,0.03)$ \\
7 & $(29,28)$ & 1050 & 16300 & 0.02 & 0.07 & $\left(0.15, \bar{\rho}_{7}, 0.23\right)$ & $\bar{\rho}_{7} \sim N(0.20,0.02)$ \\
8 & $(18,21)$ & 800 & 14900 & 0.01 & 0.06 & $\left(0.19, \bar{\rho}_{8}, 0.28\right)$ & $\bar{\rho}_{8} \sim N(0.24,0.03)$ \\
9 & $(29,23)$ & 1100 & 26500 & 0.01 & 0.06 & $\left(0.12, \bar{\rho}_{9}, 0.22\right)$ & $\bar{\rho}_{9} \sim N(0.17,0.04)$ \\
10 & $(35,26)$ & 1050 & 22000 & 0.02 & 0.05 & $\left(0.17, \bar{\rho}_{10}, 0.23\right)$ & $\bar{\rho}_{10} \sim N(0.20,0.02)$ \\
\hline
\end{tabular}

To illustrate the validity of the model and the usefulness of the solution method, the data needed to examine the CLSC performance for the four objectives is presented here. Based on the market analysis, ten coordinates for the CDC alternatives are given: location, capability, fixed costs and new product variable costs (NPV cost) and recycled product variable costs (RPV cost). These are shown in Table 2. Supermarkets and restaurants are considered to beer tailers with flexible demand. Table 3 presents the information regarding the retailers, factories and disposal centers. It can be seen from that Table $3, k_{1}$ to $k_{30}$ represents 30 different retailers, while $j_{1}$ to $j_{4}$ are the 4 different factories at different locations with variable capabilities and $n_{1}$ indicates the location and capability of the disposal center. Therefore, 30 retailers, 4 factories and 1 waste disposal center are considered in this study. The unit transportation costs and pollution are related to the distances between the facilities. The retailers' return rates are shown in Table 4, which are considered to be fuzzy random variables.

Table 3. Rtailers, factories and disposal center

\begin{tabular}{ccccccccc}
\hline Node & location & demand & Node & location & demand & Node & location & demand \\
\hline$k_{1}$ & $(27,28)$ & 50 & $k_{11}$ & $(26,39)$ & 60 & $k_{21}$ & $(25,31)$ & 70 \\
$k_{2}$ & $(30,19)$ & 60 & $k_{12}$ & $(38,26)$ & 40 & $k_{22}$ & $(29,35)$ & 90 \\
$k_{3}$ & $(32,22)$ & 40 & $k_{13}$ & $(38,34)$ & 50 & $k_{23}$ & $(18,29)$ & 50 \\
$k_{4}$ & $(37,16)$ & 80 & $k_{14}$ & $(36,25)$ & 70 & $k_{24}$ & $(18,14)$ & 60 \\
$k_{5}$ & $(23,29)$ & 30 & $k_{15}$ & $(41,19)$ & 40 & $k_{25}$ & $(35,11)$ & 80 \\
$k_{6}$ & $(27,17)$ & 40 & $k_{16}$ & $(27,33)$ & 30 & $k_{26}$ & $(23,33)$ & 50 \\
$k_{7}$ & $(33,26)$ & 80 & $k_{17}$ & $(25,39)$ & 20 & $k_{27}$ & $(36,37)$ & 60 \\
$k_{8}$ & $(34,32)$ & 40 & $k_{18}$ & $(38,37)$ & 40 & $k_{28}$ & $(28,26)$ & 40 \\
$k_{9}$ & $(37,22)$ & 100 & $k_{19}$ & $(36,27)$ & 50 & $k_{29}$ & $(25,24)$ & 30 \\
$k_{10}$ & $(17,22)$ & 90 & $k_{20}$ & $(39,28)$ & 60 & $k_{30}$ & $(32,19)$ & 80 \\
$j_{1}$ & $(13,22)$ & 920 & $j_{2}$ & $(31,44)$ & 530 & $j_{3}$ & $(32,15)$ & 850 \\
$j_{4}$ & $(42,31)$ & 940 & $n_{1}$ & $(18,47)$ & 800 & & & \\
\hline
\end{tabular}


Table 4. Retailers' return rates and CDCs' disposal rate

\begin{tabular}{cccccc}
\hline Node & $\widetilde{a}_{k}$ & Parameters $\bar{\zeta}$ & Node & $\widetilde{a}_{k}$ & Parameters $\bar{\zeta}$ \\
\hline 1 & $\left(0.28, \bar{\zeta}_{1}, 0.33\right)$ & $\bar{\zeta}_{1} \sim N(0.31,0.02)$ & 16 & $\left(0.74, \bar{\zeta}_{16}, 0.79\right)$ & $\bar{\zeta}_{16} \sim N(0.77,0.04)$ \\
2 & $\left(0.52, \bar{\zeta}_{2}, 0.62\right)$ & $\bar{\zeta}_{2} \sim N(0.56,0.02)$ & 17 & $\left(0.70, \bar{\zeta}_{17}, 0.75\right)$ & $\bar{\zeta}_{17} \sim N(0.73,0.04)$ \\
3 & $\left(0.32, \bar{\zeta}_{3}, 0.38\right)$ & $\bar{\zeta}_{3} \sim N(0.36,0.03)$ & 18 & $\left(0.75, \bar{\zeta}_{18}, 0.80\right)$ & $\bar{\zeta}_{18} \sim N(0.78,0.02)$ \\
4 & $\left(0.62, \bar{\zeta}_{4}, 0.73\right)$ & $\bar{\zeta}_{4} \sim N(0.67,0.04)$ & 19 & $\left(0.68, \bar{\zeta}_{19}, 0.75\right)$ & $\bar{\zeta}_{19} \sim N(0.72,0.03)$ \\
5 & $\left(0.55, \bar{\zeta}_{5}, 0.62\right)$ & $\bar{\zeta}_{5} \sim N(0.58,0.02)$ & 20 & $\left(0.55, \bar{\zeta}_{20}, 0.65\right)$ & $\bar{\zeta}_{20} \sim N(0.61,0.02)$ \\
6 & $\left(0.65, \bar{\zeta}_{6}, 0.72\right)$ & $\bar{\zeta}_{6} \sim N(0.69,0.02)$ & 21 & $\left(0.35, \bar{\zeta}_{21}, 0.45\right)$ & $\bar{\zeta}_{21} \sim N(0.39,0.04)$ \\
7 & $\left(0.73, \bar{\zeta}_{7}, 0.81\right)$ & $\bar{\zeta}_{7} \sim N(0.78,0.03)$ & 22 & $\left(0.35, \bar{\zeta}_{22}, 0.42\right)$ & $\bar{\zeta}_{22} \sim N(0.38,0.03)$ \\
8 & $\left(0.72, \bar{\zeta}_{8}, 0.78\right)$ & $\bar{\zeta}_{8} \sim N(0.75,0.03)$ & 23 & $\left(0.55, \bar{\zeta}_{23}, 0.60\right)$ & $\bar{\zeta}_{23} \sim N(0.58,0.03)$ \\
9 & $\left(0.75, \bar{\zeta}_{9}, 0.82\right)$ & $\bar{\zeta}_{9} \sim N(0.8,0.04)$ & 24 & $\left(0.52, \bar{\zeta}_{24}, 0.62\right)$ & $\bar{\zeta}_{24} \sim N(0.56,0.04)$ \\
10 & $\left(0.34, \bar{\zeta}_{10}, 0.38\right)$ & $\bar{\zeta}_{10} \sim N(0.36,0.02)$ & 25 & $\left(0.62, \bar{\zeta}_{25}, 0.72\right)$ & $\bar{\zeta}_{25} \sim N(0.66,0.04)$ \\
11 & $\left(0.42, \bar{\zeta}_{11}, 0.48\right)$ & $\bar{\zeta}_{11} \sim N(0.46,0.02)$ & 26 & $\left(0.69, \bar{\zeta}_{26}, 0.75\right)$ & $\bar{\zeta}_{26} \sim N(0.72,0.03)$ \\
12 & $\left(0.46, \bar{\zeta}_{12}, 0.50\right)$ & $\bar{\zeta}_{12} \sim N(0.48,0.04)$ & 27 & $\left(0.29, \bar{\zeta}_{27}, 0.35\right)$ & $\bar{\zeta}_{27} \sim N(0.32,0.03)$ \\
13 & $\left(0.65, \bar{\zeta}_{13}, 0.70\right)$ & $\bar{\zeta}_{13} \sim N(0.67,0.04)$ & 28 & $\left(0.40, \bar{\zeta}_{28}, 0.46\right)$ & $\bar{\zeta}_{28} \sim N(0.43,0.02)$ \\
14 & $\left(0.62, \bar{\zeta}_{14}, 0.68\right)$ & $\bar{\zeta}_{14} \sim N(0.64,0.03)$ & 29 & $\left(0.42, \bar{\zeta}_{29}, 0.48\right)$ & $\bar{\zeta}_{29} \sim N(0.45,0.03)$ \\
15 & $\left(0.72, \bar{\zeta}_{15}, 0.78\right)$ & $\bar{\zeta}_{15} \sim N(0.75,0.04)$ & 30 & $\left(0.50, \bar{\zeta}_{30}, 0.58\right)$ & $\bar{\zeta}_{30} \sim N(0.54,0.04)$ \\
\hline
\end{tabular}

\subsection{Sensitivity analysis on the parameters}

To find the best solution to the proposed model, a series of experiments were conducted, all of which were performed using a MATLAB 7.0 on a workstation with an Intel(R) Corei5, a Pentium 4, $1.83 \mathrm{GHz}$ clock pulse with 4GB memory and Windows 10 operating system. A sensitivity analysis was performed to exhibit the effectiveness and behavior of the proposed algorithm, as shown in Table 5. Several parameters were changed, including the population size $N$, maximum generation $T$ and acceleration constant $c_{p}, c_{g}, c_{l}$ and $c_{n}$. After trying various values for the population size and maximum generations, the results were found to be better when $T$ was from 200 to 400 and $N$ was from 30 to 50. The different fitness values obtained using the pb-glnPSO with the different parameters $N T c_{1}$ and $c_{2}$ are shown in Table 5.

Table 5. Sensitivity analysis (unit: $1 \times 10^{2} \mathrm{RMB}$ )

\begin{tabular}{|c|c|c|c|c|c|c|c|c|c|}
\hline & $T=200$ & $T=300$ & $T=400$ & $T=200$ & $T=300$ & $T=400$ & $T=200$ & $T=300$ & $T=400$ \\
\hline 0.5 & 88049.270 & 85447.261 & 86943.479 & 89889.371 & 88266.237 & 84859.596 & 86479.923 & 84830.640 & 88233.839 \\
\hline 1 & 87056.401 & 84125.454 & 84428.258 & 88679.314 & 87459.033 & 84199.470 & 85596.767 & 83448.794 & 85614.352 \\
\hline 1.5 & 86601.808 & 84465.348 & 84315.569 & 85486.560 & 86564.333 & 84041.957 & 84698.625 & 83257.776 & 82930.618 \\
\hline 2 & 85614.087 & 82308.935 & 83058.532 & 82914.087 & 82434.420 & 83681.801 & 82884.954 & 81920.133 & 82585.367 \\
\hline 2.5 & 85844.692 & 83102.944 & 87782.377 & 85170.526 & 83198.452 & 86792.664 & 83448.789 & 84469.925 & 83177.298 \\
\hline
\end{tabular}

As can be seen from Table 5, when the parameters $c_{p}, c_{g}, c_{l}$ and $c_{n}$ increase, the fitness value improves except for $c_{p}=c_{g}=c_{l}=c_{n}=2.5$ with the same generation and popsize, with the fitness value increasing from $c_{p}=c_{g}=c_{l}=c_{n}=2$ to $c_{p}=c_{g}=c_{l}=c_{n}=2.5$. Therefore, when $c_{p}=c_{g}=c_{l}=c_{n}=2$, the result is optimal. For $T$, given the same $c_{p}, c_{g}, c_{l}$ and $c_{n}$ and population size, the results shows that when $T$ is 300 , the fitness value is better than for any other generation. Finally, for $N$, the results improve as the population size increases and is optimal when $N$ is 50 . The most effective and efficient results are gained with $T$ at $300, N$ at 50 and $c_{p}=c_{g}=c_{l}=c_{n}=2$.

\subsection{Result analysis}

In this section, the pb-glnPSO is performed to solve the model using the above data. The parameters for the problem were set as follows: Population size: popsize $=50$; Maximum generation: $\operatorname{maxGen}=300$; Inertia weight: $\omega(1)=1$ and $\omega(T)=0.1$; Acceleration constant: $c_{p}=c_{g}=c_{l}=c_{n}=2$. After running the program 20 times, the best satisfactory solution was found. Figure 4 shows the specific objective values found by the $\mathrm{Pb}$-glnPSO in different iterations and shows the reductions in the total 


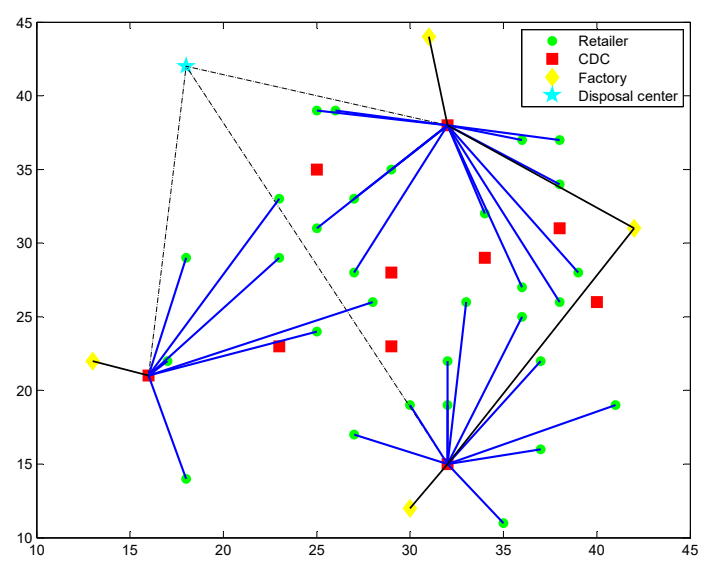

Figure 3. The distribution strategy

costs. The results are presented in Table 6 and Figure 3. From the calculation, at least 3 CDCs could satisfy all markets. The result show that alternative CDC positions 4,5 and 8 should be chosen as CDC 4 can send products to markets $2,3,4,6,7,9,14,15,25,30$, CDC 5 can transport products for 1, 8, 11, $12,13,16,17,18,19,20,21,22,27$ and retailers 5, 10, 23, 24, 26, 28, 29 can be serviced by CDC 8 . The total cost is 81.936 million RMB, in which the fixed costs are 44 million RMB, the transportation costs are 37.759 million RMB and the operating costs are 17.7 million RMB.

Table 6. Results

\begin{tabular}{|c|c|c|c|c|c|c|c|c|c|c|c|}
\hline \multicolumn{2}{|c|}{ Factories } & \multicolumn{2}{|c|}{ DR-centers } & \multicolumn{2}{|c|}{ Markets } & \multicolumn{2}{|c|}{ Factories } & \multicolumn{2}{|c|}{ DR-centers } & \multicolumn{2}{|c|}{ Markets } \\
\hline Node & location & Node & location & Node & location & Node & location & Node & location & Node & location \\
\hline & & & & 5 & $(23,29)$ & & & & & 1 & $(27,28)$ \\
\hline & & & & 10 & $(17,22)$ & & & & & 8 & $(34,32)$ \\
\hline & & & & 23 & $(18,29)$ & & & & & 11 & $(26,39)$ \\
\hline \multirow[t]{7}{*}{1} & $(13,22)$ & 8 & $(18,21)$ & 24 & $(18,14)$ & 2 & $(31,44)$ & 5 & $(35,37)$ & 12 & $(38,26)$ \\
\hline & & & & 26 & $(23,33)$ & & & & & 13 & $(38,34)$ \\
\hline & & & & 28 & $(28,26)$ & & & & & 16 & $(27,33)$ \\
\hline & & & & 29 & $(25,24)$ & & & & & 17 & $(25,39)$ \\
\hline & & & & 2 & $(30,1$ & & & & & 25 & $(35,11)$ \\
\hline & & & & 3 & $(32,22)$ & & & 4 & $(32,25)$ & 30 & $(32,19)$ \\
\hline & & & & 4 & $(37,16)$ & & & & & 18 & $(38,37)$ \\
\hline \multirow{5}{*}{3} & & & & 6 & $(27,17)$ & & & & & 19 & $(36,27)$ \\
\hline & $(32,15)$ & 4 & $(32,25)$ & 7 & $(33,26)$ & 4 & $(42,31)$ & & & 20 & $(39,28)$ \\
\hline & & & & 9 & $(37,22)$ & & & & & 21 & $(25,31)$ \\
\hline & & & & 14 & $(36,25)$ & & & 5 & $(35,37)$ & 22 & $(29,35)$ \\
\hline & & & & 15 & $(41,19)$ & & & & & 27 & $(36,37)$ \\
\hline
\end{tabular}

\subsection{Algorithm comparison}

To better illustrate the effectiveness of the proposed algorithm, a brief comparison between the pb-glnPSO, glnPSO and an immune algorithm(IM) is given in this section. The glnPSO is a well-respected evolutionary algorithm and has been successfully implemented in a variety of engineering and combinatorial problems. The IM has also being widely used to solve facilities location problems.

To establish the solution quality for the pb-glnPSO, it is compared with the glnPSO and the IM. Each run time for the pb-glnPSO, glnPSO and the IM was around 80s. The pb-glnPSO, glnPSO and IM were run 20 times using the same data. For a fair comparison between the groups, each population with the same number was initialized with the population size set at50 and the maximum generation at 300. In the glnPSO, an acceleration constant was designed as $c_{p}=c_{g}=c_{l}=c_{n}=2$ and the inertia weight 
was $\omega(1)=1$ and $\omega(T)=0.1$. For the IM algorithm, the crossover probability was 1 and the mutation probability was 0.1 .
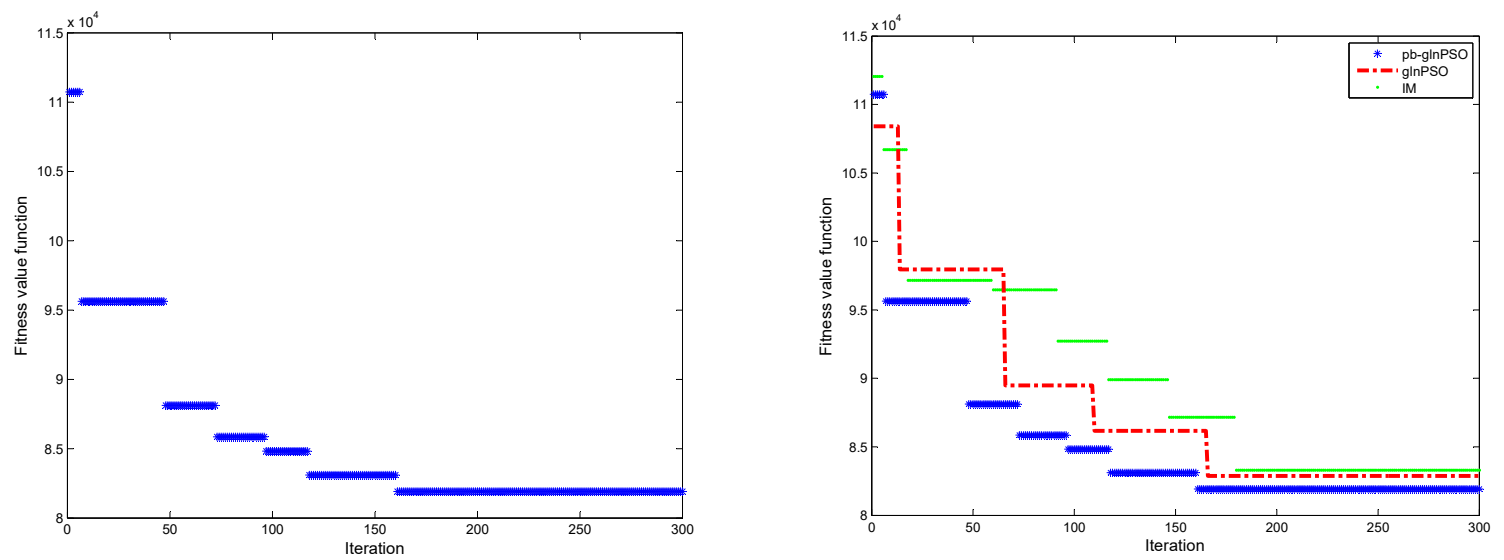

Figure 4. The iterative process of pb-glnPSO,glnPSO and IM

From Figure 4, it can be seen that the pb-glnPSO outperformed both the glnPSO and the IM, and as the glnPSO converged faster, it had a better result than the IM. This demonstrates that a better solution can be obtained using the glnPSO, and especially using the pb-glnPSO. The blue profile shows the convergence for the best in history for the pb-glnPSO. It can be seen from Figure 4 that as the programs ran, the results become stable for the pb-glnPSO and glnPSO after about the 160th generation, while the IM became stable after the 180th generation. As is shown in Figure 4, the best solution for the pb-glnPSO was superior to, more stable than and had the smallest CPU run time than the other algorithms( Table 7), with the IM having the highest run time.

Table 7. Results of the pb-glnPSO, glnPSO, and IM

\begin{tabular}{lccc}
\hline Item & Pb-glnPSO & glnPSO & IM \\
\hline Best result & 81920.133 & 82884.954 & 83315.569 \\
Worst result & 83102.944 & 84826.640 & 85585.473 \\
Average result & 82431.119 & 83877.688 & 84179.884 \\
Difference between the best and the worst & 1182.811 & 1941.686 & 2269.904 \\
Difference between the average and the best & 510.986 & 992.734 & 864.315 \\
Standard deviation & 317.467 & 553.194 & 680.326 \\
CPU time & 88.7969 & 124.9844 & 161.5000 \\
\hline
\end{tabular}

\section{Conclusion}

Economic development has caused many environmental pollution problems,the seriousness of which has encouraged people to recycle and reuse products. To examine this problem and seek appropriate solutions, a multi-objective collection-distribution center location and allocation problem in a closed loop supply chain under a fuzzy random environment was presented in this paper for the beer industry in China. For this problem, a new model was formulated, in which the decision makers sought to minimize costs and pollution underflow, capability and quantity limit constraints. To more accurately represent actual production situations, the return rate and disposal rate were considered fuzzy random variables. A heuristic algorithm, the pb-glnPSO, was then applied to solve the problem. Based on the proposed priority, the distribution and collection activity was shown to satisfy retailer demand, and reduce costs and pollution after the CDCs started operations. After calculation, the best solution was determined and the advantages of the algorithm illustrated. The proposed model and method can be applied for the location and allocation of CDCs in the beer industry, assisting in improving effective supply chain management. The model was shown to assist in generating retailer demand and dealing with the returned products first, which could benefit company recycling and 
reuse policies. At the same time, the transportation costs and pollution were reduced because of the reduction in losses from empty loads.

\section{References}

1. Li, J.; Du, W.; Yang, F.; Hua, G. The Carbon Subsidy Analysis in Remanufacturing Closed-Loop Supply Chain. Sustainability 2014, 6(6), 3861-3877.

2. Ma, J.; Wang, H. Complexity analysis of dynamic noncooperative game models for closed-loop supply chain with product recovery. Applied Mathematical Modelling 2014, 38(23), 5562-5572.

3. Giri, B.C.; Sharma, S. Optimizing a closed-loop supply chain with manufacturing defects and quality dependent return rate. Journal of Manufacturing Systems 2015, 35, 92-111.

4. Rezapour, S.; Farahani, R.Z.; Fahimnia, B.; Govindan, K.; Mansouri, Y. Competitive closed-loop supply chain network design with price-dependent demands. Journal of Cleaner Production 2015, 93, 1-22.

5. Subramanian, P.; Ramkumar, N.; Narendran, T.T. PRISM: PRIority based SiMulated annealing for a closed loop supply chain network design problem. Applied Soft Computing 2013, 13, 1121-1135.

6. Amin, S.H; Zhang, G. A multi-objective facility location model for closed-loop supply chain network under uncertain demand and return. Applied Mathematical Modelling 2013, 37(6), 4165-4176.

7. Subulan, K.; Baykasoğlu, A.; Özsoydan, F.B.; Taşan, A.S; Selim, H. A case-oriented approach to a lead/acid battery closed-loop supply chain network design under risk and uncertainty. Journal of Manufacturing Systems 2014, 37, 340-361.

8. Zeballos, L.J.; Méndez, C.A.; Barbosa-Povoa, A.P.; Novais, A.Q. Multi-period design and planning of closed-loop supply chains with uncertain supply and demand. Computers \& Chemical Engineering 2014, 66(27), 151-164.

9. Oh, J.; Jeong, B. Profit analysis and supply chain planning model for closed-loop supply chain in fashion industry. Sustainability 2014, 6(12), 9027-9056.

10. Khatami, M.; Mahootchi, M.; Farahani, R.Z. Benders' decomposition for concurrent redesign of forward and closed-loop supply chain network with demand and return uncertainties. Transportation Research Part E Logistics \& Transportation Review 2015, 79, 1-21.

11. Vahdani, B.; Mohammadi, M. A bi-objective interval-stochastic robust optimization model for designing closed loop supply chain network with multi-priority queuing system. International Journal of Production Economics 2015, 170, 67-87.

12. Kim, S.; Jeong, B. Closed-Loop Supply Chain Planning Model for a Photovoltaic System Manufacturer with Internal and External Recycling. Sustainability 2016, 8(7).

13. Kaya, O.; Urek, B. A mixed integer nonlinear programming model and heuristic solutions for location, inventory and pricing decisions in a closed loop supply chain. Computers \& Operations Research 2015, 65(C), 93-103.

14. Ramkumar, N.; Subramanian, P.; Narendran, T.T.; Ganesh, K. A genetic algorithm approach for solving a closed loop supply chain model: A case of battery recycling Applied Mathematical Modelling 2011, 34(3), 655-670.

15. Barz, A.; Buer, T.; Haasis, H.D. Quantifying the effects of additive manufacturing on supply networks by means of a facility location-allocation model. Bremen Computational Logistics Group Working Papers 2015, 9, $1-14$.

16. Jindal, A.; Sangwan, K.S. Multi-objective fuzzy mathematical modelling of closed-loop supply chain considering economical and environmental factors. Annals of Operations Research 2016, 1-26.

17. Ramezani, M.; Kimiagari, A.M.; Karimi, B.; Hejazi, T.H. Closed-loop supply chain network design under a fuzzy environment. Knowledge-Based Systems 2014, 59, 108-120.

18. Eleonora, B.; Roberto M.; Marta, R.; Giuseppe V. Modeling and multi-objective optimization of closed loop supply chain: A case study. Computer $\mathcal{E}$ Industrial Engineering 2015, 87, 328-342.

19. Fallah, H.; Eskandari, H.; Pishvaee, M. Competitive closed-loop supply chain network design under uncertainty. Journal of Manufacturing Systems 2015, 37, 649-661.

20. Zhalechian, M.; Tavakkoli-Moghaddam, R.; Zahiri, B; Mohammadi, M. Sustainable design of a closed-loop location-routing-inventory supply chain network under mixed uncertainty. Transportation Research Part E: Logistics and Transportation Review 2016, 89, 182-214. 
21. Hatefi, S.M.; Jolai, F.; Torabi, S.A.; Tavakkoli-Moghaddam, R. Reliable design of an integrated forward-revere logistics network under uncertainty and facility disruptions: A fuzzy possibilistic programing model. Ksce Journal of Civil Engineering 2015, 19, 1-12.

22. Zhong, S.; Chen, Y.; Zhou, J. Fuzzy random programming models for location-allocation problem with applications. Computers $\mathcal{E}$ Industrial Engineering 2014, 89, 194-202.

23. Keyvanshokooh, E.; Ryan, S.M.; Kabir, E. Hybrid robust and stochastic optimization for closed-loop supply chain network design using accelerated Benders decomposition. European Journal of Operational Research 2016, 249, 76-92.

24. Ma, Y.; Xu, J. A novel multiple decision-maker model for resource-constrained project scheduling problems. Canadian Journal of Civil Engineering 2014, 41, 500-511.

25. Ma, Y.; $\mathrm{Xu}, \mathrm{J}$. Vehicle routing problem with multiple decision-makers for construction material transportation in a fuzzy random environment. International Journal of Civil Engineering 2014, 12, 332-346.

26. Maghsoudlou, H.; Kahag, M.R.; Niaki, S.T.A.; Pourvaziri, H. Bi-objective optimization of a three-echelon multi-server supply-chain problem in congested systems: Modeling and solution. Computers $\mathcal{E}$ Industrial Engineering 2016, 99, 41-62.

27. Xu, J.; Ma, Y.; Xu, Z. A Bilevel Model for Project Scheduling in a Fuzzy Random Environment. IEEE Transactions on Systems, Man, and Cybernetics: Systems 2015, 45, 1322-1335.

28. Li, B.; Wang, L.; Liu, B. An Effective PSO-Based Hybrid Algorithm for Multiobjective Permutation Flow Shop Scheduling. Systems Man \& Cybernetics Part A Systems \& Humans IEEE Transactions on 2008, 38, 818-831.

29. Chen, T.; Chi, T. On the improvements of the particle swarm optimization algorithm. Advances in Engineering Software 2010, 41, 229-239.

30. Ai, T.J.; Kachitvichyanuku, V. A particle swarm optimization for the vehicle routing problem with simultaneous pickup and delivery. Computers $\mathcal{E}$ Operations Research 2009, 36, 1693-1702.

31. Zhang, Z.; Wang, Z.; Liu, L.; Retail Services and Pricing Decisions in a Closed-Loop Supply Chain with Remanufacturing. Sustainability 2015, 7, 2373-2396.

32. Huynh, C.H.; So,K.C.; Gurnani, H. Managing a Closed-Loop Supply System with Random Returns and a Cyclic Delivery Schedule. European Journal of Operational Research 2016, 255, 787-796.

33. Zohal, M.; Soleimani, H. Developing an ant colony approach for green closed-loop supply chain network design: a case study in gold industry. Journal of Cleaner Production 2016, 133, 314-337.

34. Zhang, Z.; Unnikrishnan, A. A coordinated location-inventory problem in closed-loop supply chain. Transportation Research Part B Methodological 2016, 89, 127-148.

35. Kruse, R.; Meyer, K. Statistics with Vague Data 1987. Reidel Publishing Company.

36. Heilpern,S. The expected value of a fuzzy number. Fuzzy Sets and Systems 1992, 47, 81-86.

37. Talaei, M.; Moghaddam, B.F.; Pishvaee, M.S.; Bozorgi-Amiri, A.; Gholamnejad, S. A robust fuzzy optimization model for carbon-efficient closed-loop supply chain network design problem: a numerical illustration in electronics industry. Journal of Cleaner Production 2016, 113, 662-673.

38. Kennedy, J.; Eberhart, R.C. Particle swarm optimization. Journal Abbreviation 1995, 4, 1942-1948.

39. Soleimani, H.; Kannan, G. A hybrid particle swarm optimization and genetic algorithm for closed-loop supply chain network design in large-scale networks. Applied Mathematical Modelling 2015, 39, 3990-4012.

40. Xu, J.; Yan, F.; Li, S. Vehicle routing optimization with soft time windows in a fuzzy random environment. Transportation Research Part E Logistics E Transportation Review 2011, 47, 1075-1091.

41. Gen, M.; Altiparmak, F.; Lin, L. A genetic algorithm for two-stage transportation problem using priority-based encoding. OR spectrum 2006, 28, 337-354.

Sample Availability: Samples of the compounds ...... are available from the authors.

(C) 2016 by the authors; licensee Preprints, Basel, Switzerland. This article is an open access article distributed under the terms and conditions of the Creative Commons Attribution (CC-BY) license (http://creativecommons.org/licenses/by/4.0/). 